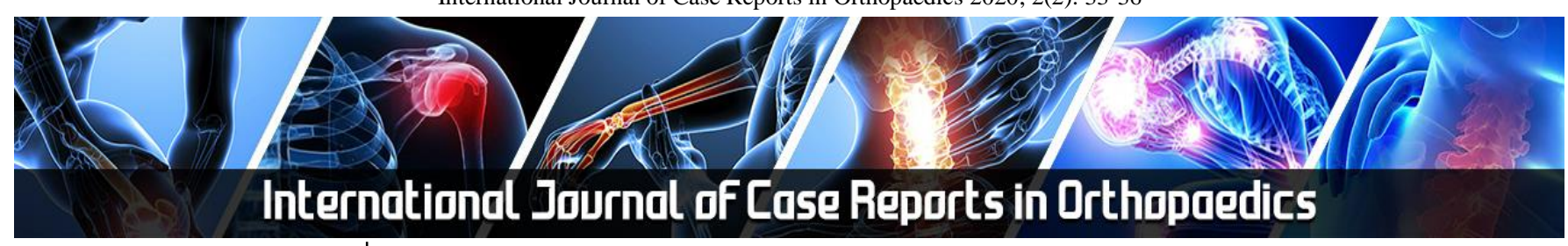

E-ISSN: 2707-8353 P-ISSN: 2707-8345 IJCRO 2020; 2(2): 33-36 Received: 16-04-2020 Accepted: 03-06-2020

Ishan Shevate

MBBS, MS, DNB

Orthopaedics, MRCS (Edin), Assistant Professor,

Department of Orthopaedics, Dr. D.Y. Patil Medical College, Hospital and Research Centre, Pune, Maharashtra, India

Ashwinkumar Khandge MBBS, MS, DNB

Orthopaedics, Assistant

Professor, Department of Orthopaedics, Dr. D.Y. Patil Medical College, Hospital and Research Centre, Pune,

Maharashtra, India

Girish Nathani

MBBS, MS Orthopaedics, Senior Resident, Department of Orthopaedics, Dr. D.Y. Patil Medical College, Hospital and Research Centre, Pune, Maharashtra, India

Aanchal Mal MBBS, Department of Orthopaedics, Dr. D.Y. Patil Medical College, Hospital and Research, Centre, Pune, Maharashtra, India
Corresponding Author: Ishan Shevate MBBS, MS, DNB Orthopaedics, MRCS (Edin), Assistant Professor, Department of Orthopaedics, Dr. D.Y. Patil Medical College, Hospital and Research Centre, Pune, Maharashtra, India

\section{Tuberculosis of talus with ankle joint involvement: A case report}

\author{
Ishan Shevate, Ashwinkumar Khandge, Girish Nathani and Aanchal \\ Mal
}

DOI: https://doi.org/10.22271/27078345.2020.v2.i2a.27

\begin{abstract}
Case: We present a case of a 17-years-old lady, with right ankle joint pain and swelling. The diagnosis of tuberculosis of the talus with involvement of the distal tibia and ankle joint was made with the help of X-rays, MRI, gene expert and core needle biopsy from the talus. She was treated with anti-tubercular chemotherapy and ankle immobilisation.

Conclusion: Early diagnosis with radiological studies, gene expert and histopathology will help preventing further destruction of the joint surfaces due to this rare form of skeletal tuberculosis. This along with a conservative treatment with anti-tubercular drugs and protected weight bearing gives excellent functional results.
\end{abstract}

Keywords: Tuberculosis and Ankle Joint

\section{Introduction}

Tuberculosis (TB) still remains one of the most common cause of morbidity and mortality in developing parts of the world ${ }^{[1]}$. Extra pulmonary involvement is seen in $23-30 \%$ of TB cases. The musculoskeletal system is affected in 1-2\% of cases, mainly involving the spine and large weight bearing joints of lower extremity such as hip and knee ${ }^{[2,3]}$. The incidence of foot and ankle tuberculosis is only $1 \%$ of all tubercular infections ${ }^{[4,5,6]}$. Tuberculosis of talus with ankle joint involvement is very rarely reported so far in the literature. Calcaneum being the most commonly affected bone ${ }^{[4]}$.

Often the diagnosis of these rare osteoarticular tuberculosis cases gets delayed on clinical basis because of atypical symptomatology and normal chemistries in most of the cases [7]. This leads to delayed diagnosis and treatment, resulting in poorer outcome in the form of affection of adjacent bones and joint destruction. Prompt diagnosis and management is important which depends upon high index of suspicion and awareness about the atypical clinical and radiological signs. The special tests for tuberculosis help to arrive at an early diagnosis. Here we discuss a case of a young lady suffering from TB of right talus with ankle joint extension treated conservatively with antitubercular medications, immobilization of the ankle joint and protected weight-bearing giving an excellent functional outcome.

\section{Case Report}

A 16 years-old female child came to the orthopaedic OPD with a 4 months history of pain and swelling in the right ankle region. There was history of trauma 4 months back, when the patient twisted her right ankle after placing her foot in a ditch, there was no external injury. The patient also complained of difficulty in walking and ankle movements. There was no history of fever, night sweats, weight loss associated. The general examination was within normal limits.

On local examination, the temperature was raised as compared to the leg, there was tenderness over the antero-medial and anterior aspect of the ankle joint. Localised swelling was present on the antero-medial aspect. It was approximately $5 \mathrm{~cm} \times 6 \mathrm{~cm}$, ovoid in shape with well-defined regular margins. Overlying skin was tense and the swelling was fixed to the bone and immobile. Painful restriction of movements was demonstrated with dorsiflexion and plantar flexion $0^{\circ}$ to $10^{\circ}$.

Serological examination demonstrated reduced haemoglobin $(\mathrm{Hb})$ of $8.5 \mathrm{gm} / \mathrm{dl}$, reduced red blood cell (RBC) count of 3.65 million, white blood cell (WBC) count of 11,600, an elevated erythrocyte sedimentation rate (ESR) of $65 \mathrm{~mm}$ per hour (Westergren method), a positive C- 
reactive protein (CRP) test. The mantoux test was negative. The chest $X$ rays were normal. Radiographs of the ankle were obtained in 3 orthogonal planes: anteroposterioir (AP), mortise and lateral. It showed a solitary, localised, irregular, lytic lesion with sclerotic margins in the body and head of talus without any signs of involvement of the adjacent bones.

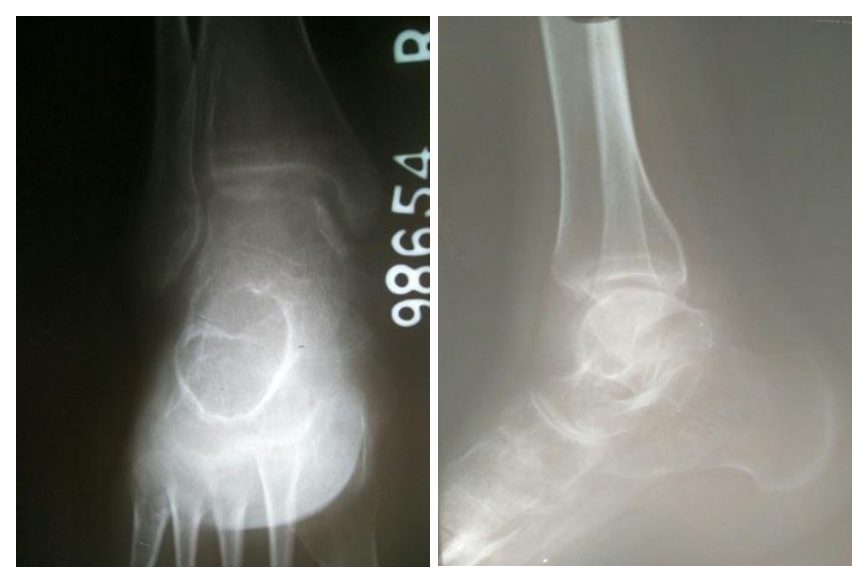

Pre-operative X-ray images

The Magnetic Resonance Imaging showed a peripherally enhancing intraosseous abscess in medial portion of the head of talus measuring $20 \mathrm{~mm} \times 12 \mathrm{~mm} \times 12 \mathrm{~mm}$ with perilesional marrow oedema. Cortical discontinuity along the medial aspect with extension of the abscess into the synovial cavity was present. Thickening and enhancement of the talo-crural joint with associated effusion due to synovitis was seen. Minimal marrow oedema was seen in the subarticular region of distal tibia.

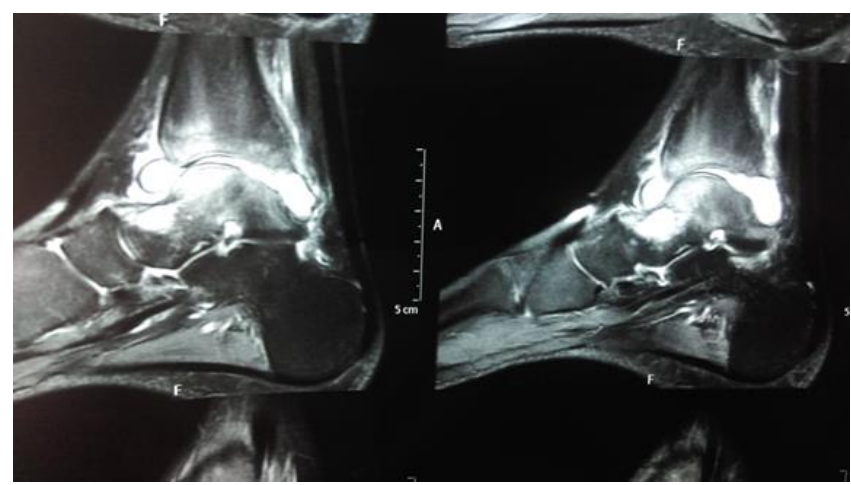

Pre-operative MRI images

After taking informed consent from the patient, core needle biopsy was taken from the lesion under local anaesthesia using Image Intensifier guidance with the help of $\mathbf{J}$ (Jamshedi) needle. On histopathological examination, the biopsy tissue showed epitheloid cell granulomas with central caseating necrosis, with Langhans' type giant cells. The biopsy sample was also tested for acid fast bacilli staining, which came out negative. The sample was further sent for BACTEC culture and CBNAAT/gene expert testing. Their reports confirmed mycobacterium tuberculosis. After we got the tissue diagnosis confirmation Anti-Tubercular chemotherapy was initiated, which included 4 drugs Isoniazid (INH), Rifampicin, Pyrazinamide and Ethambutol for the intensive phase of 3 months period, which was followed by 2 drugs continuation regimen of INH and Rifampicin for the subsequent 9 months. The ankle joint was immobilised using a plaster of Paris (POP) boot cast in neutral position for 12 weeks. During this time patient was kept on non-weight bearing walking with walker support. At 12 weeks follow up $\mathrm{X}$ rays showed signs of early healing. CBC along with ESR was done, which showed a declining trend. The cast was removed at this point and ankle physiotherapy started. Weight bearing was allowed gradually and according to the pain tolerance in an Air cast. X rays and blood parameters were repeated at 6, 9 and 12 months. The patient obtained a painless range of ankle motion and could do full weight bearing walking at 1 year follow up after finishing the 12 months chemotherapy. At 1 year, follow up X rays of right ankle in AP and lateral views demonstrated excellent bone healing of the osteolytic lesion in the talus with no signs of arthritis in the ankle joint.

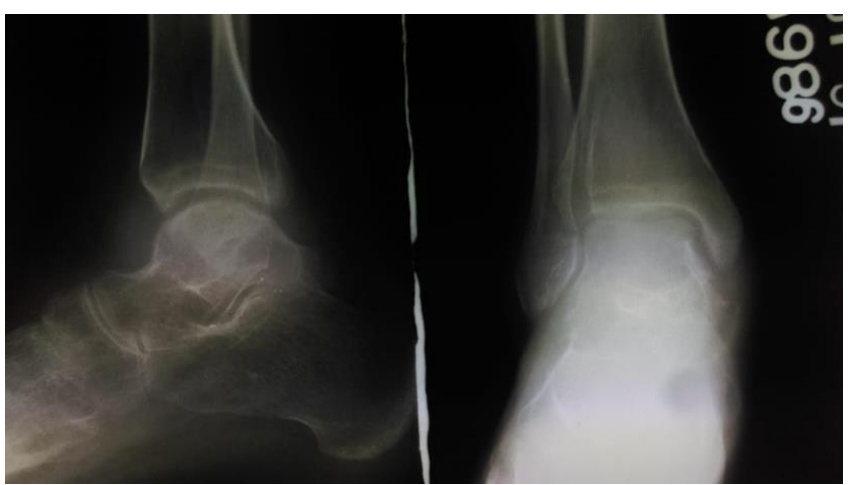

Healed X-ray images

\section{Discussion}

Haraldsson reported a case of talus TB in a two-and-halfyear-old child following administration of Bacillus Calmette-Guérin (BCG) vaccine. Due to lack of awareness and clinical suspicion owing to its rarity, there was a delay of five weeks in the correct diagnosis of isolated talus TB in a six-year-old child. Goksan, et al. reported conservative treatment with a combination of three drug chemotherapy for a case of tuberculous osteitis of the talus in a nine-yearold child. Jockheck, et al. treated a Vietnamese patient with tubercular osteitis of the talus with partial astragalectomy along with tibio-calcaneal fusion using the Charnley fixator. In another report of isolated talus TB, Boussouga, et al. treated a 66-year-old patient conservatively with three drug anti-TB chemotherapy. In the above case, standard laboratory investigations, as well as imaging studies like MRI and X-ray, failed to establish the diagnosis of TB, which was done by histopathological examination ${ }^{[5]}$.

Anand and Sood reported operative treatment with curettage and bone grafting in a case of the osteolytic type of talar TB in an eight-year-old child with unremarkable routine blood investigations, erythrocyte sedimentation rate (ESR) and Mantoux test. In a retrospective analysis of the foot and ankle TB cases, Dhillon, et al. found only a single case of talus TB out of 992-foot cases over a period of 20 years. Dahuja, et al. reported a case of isolated TB of the talus treated with surgical curettage and debridement along with anti-TB chemotherapy giving good results. Halwai, et al. reported the use of transmalleolar osteotomy for surgical debridement and obtaining tissue samples in a talar TB case who had a normal ankle joint range of motion after six years [6]. 


\section{Discussion}

Tuberculosis is a very common infection in developing countries like India leading to significant impairment and ultimately death ${ }^{[8]}$. Plantar region affection by the disease is a relatively rare occasion ${ }^{[1]}$. Atypical clinical presentation of this rare and occult type of skeletal Tuberculosis makes it a difficult lesion to diagnose early and treat ${ }^{[7]}$. Various radiological investigation like X-ray, CECT, MRI can be done to identify and confirm the diagnosis. However, these tests are non-specific and can mimic other diseases like septic arthritis ${ }^{[5,11]}$. The confirmation of disease can be only made by isolation of the organism from the lesion which can be done by Ziel Nelson Staining, microscopy of growth of mycobacteria on liquid culture and histopathological examination. Newer techniques like BACTEC MGIT liquid culture, Cartridge Based Nucleic Acid Amplification Test (CB-NAAT)/Gene Xpert are used for quick confirmation of the disease in less than a day and also tells us about the resistance to first line anti tubercular drugs likes rifampicin. In our case we arrived at a timely diagnosis inspite of vague symptoms and serological picture due to the Xpert/CBNAAT (Cartridge based Nucleic Acid Amplification Test) testing of the biopsy tissue. Literature collectively shows that Xpert MTB/CB-NAAT provides a rapid diagnosis in approximately $50-80 \%$ of extra pulmonary $\mathrm{TB}$ cases in a majority of studies ${ }^{[9]}$. CB-NAAT is a highly sensitive and specific test with less time consumption for early detection of Extra-Pulmonary Tuberculosis (EPTB) ${ }^{[10]}$.

Various treatment options have been discussed in the literature. Conservative treatment in a paediatric case of isolated talus tuberculosis with three drug chemotherapy was reported by Goksan et al. ${ }^{[12]}$. Haraldsson et al. presented a case of TB talus in a young child following Bacillus Calmette-Guérin (BCG) vaccination ${ }^{[14]}$. Anderson et al. discussed a case of six year old child with delayed diagnosis of isolated talus TB due to the lack of suspicion and knowledge about the disease ${ }^{[5]}$. Jockheck et al. managed a case of talus tuberculosis with partial talectomy and tibio-calcaneal arthrodesis with Charnleys external fixtior ${ }^{[2]}$. Boussouga et al. described a case in which routine blood and radiological investigations failed to diagnose the infection which was made by histopathological examination and further conservatively treated the patient with 3 drug chemotherapy ${ }^{[1]}$. Anand et al. reported a case in 8 years old child with insignificant blood parameters and ESR. They did curettage and bone grafting of the osteolytic talar lesion ${ }^{[13]}$. Surgical curettage and debridement followed with postoperative anti tubercular drugs gave good results in a study conducted by Dahuja et al. ${ }^{[8]}$. Dhillon et al. in a retrospective study of over 20 years of foot and ankle TB cases found only a single case of talus TB out of 992 cases. Halwai et al. did trans malleolar osteotomy and surgical debridement for talus tuberculosis ${ }^{[15]}$. Karkhur et al. presented a case of a young male with isolated TB of right talus treated conservatively with anti-TB drugs, immobilization of the ankle joint and protected weightbearing with good functional outcome ${ }^{[16]}$.

A detailed knowledge about this rare presentation of skeletal Tuberculosis would help surgeons to have an high index of suspicion and keeping it as a differential diagnosis in all the inflammatory and infective affections of foot and ankle. Delayed diagnosis in such patients results in a high degree of morbidity. In our case MRI, histopathological diagnosis and Gene Xpert helped in arriving at an accurate diagnosis in considerably less time. Diagnosis at an early stage and starting a conservative treatment with a prolonged antitubercular chemotherapy as in any other osteoarticular TB and immobilisation yeilded good results. Depending on the stage of the disease and response to chemotherapy surgical management in the form of debridement, talectomy with talo-calcaneal arthrodesis can be done. Further studies are needed to ascertain the sensitivity and specificity of the recently developed investigations in the diagnosis of foot and ankle tuberculosis.

\section{Conclusion}

Along with a high index of suspicion, histopathological examination and Gene Xpert test on the biopsy tissue we could arrive at an early diagnosis of this rare and occult type of skeletal Tuberculosis. This led us to start prompt treatment of the infection with one year of anti-tubercular drugs, ankle joint immobilisation and protected weight bearing lead to complete resolution of the disease with excellent functional and radiological outcome. Thus, preventing spread of the infection to the adjoining bones, joints and surrounding soft tissues.

Hence early diagnosis of this rare lesion and timely management in the early stages of the disease can prevent the need of surgery and gives good function.

\section{References}

1. Boussouga M, Tanane M, Bousselmame N, Lazrak K, Taobane H. [Tuberculosis of the talus: a rare localization of Koch's bacillus] Revue de Chirurgie Orthopedique et Reparatrice de L'appareil Moteur 2002;88(5):522-525. [PubMed] [Google Scholar]

2. Jockheck M, Meeder PJ, Weller S. Die tuberkulöse Destruktion des Talus--Eine seltene form der extrapulmonalen Skelett-TBC--Eine Fallbeschreibung [Tuberculous destruction of the talus-a rare form of extrapulmonary skeletal TBC-a case report]. Z Orthop Ihre Grenzgeb 1992;130(2):163-165.[PubMed] [Google Scholar]

3. Tuli SM. Tuberculosis of the skeletal system. 3rd ed. New Delhi: Jaypee Brothers 2004, 124-134.

4. Dhillon MS, Aggarwal S, Prabhakar S, Bachhal V. Tuberculosis of the foot: An osteolytic variety. Indian J Orthop 2012;46(2):206-211.

5. Anderson JR, Ritchie AW, Scobie WG. Tuberculous osteitis of the talus. Tubercle 1979;60(2):115-118. [PubMed] [Google Scholar]

6. Ruggieri M, Pavone V, Polizzi A, et al. Tuberculosis of the ankle in childhood: clinical, roentgengraphic and computed tomography findings. Clin Pediart (Phila) 1997;36:529-534.

7. Khan FA, Khoshhlal K, Saadeddin M. Tuberculosis of talus and cuboid-a report of 2 children. Acta Orthop Scand 1999;70:637-39.

8. Dahuja A, Dahuja G, Kaur R, et al. Isolated tuberculosis of talus: a case report. Malays Orthop J 2014;8:61-2. 10.5704/MOJ.1403.001

9. Lawn SD, Zumla AI. Diagnosis of extrapulmonary tuberculosis using the Xpert@ MTB/RIF assay. Expert Rev Anti Infect Ther 2012;10(6):631-635.

10. Daksh Sharma, Sanjay Tandon, Nagdeote ST, Vishal Patidar. Diagnostic Accuracy of CBNAAT in Spinal Tuberculosis. People's Journal of Scientific Research 2018;11(1):42-47. 
11. Mittal R, Gupta V, Rastogi V. Tuberculosis of the foot. J Bone Joint Surg Br 1999;81:997-1001.

12. Göksan A, Yazicioglu $\mathrm{O}$, Ozger $\mathrm{H}$, et al. Isolated bone tuberculosis of the talus. A case report [article in German-English]. Z Orthop Unfall 1984;122:657-8. 10.1055/s-2008-1045046.

13. Anand A, Sood LK. Isolated tuberculosis of talus without ankle and subtalar joint involvement Med J Malaysia 2002;57:371-373.

14. Haraldsson S. Osteitis tuberculosa fistulosa following vaccination with E.C.G. strain. Acta Orthop Scand 1959;29:121-33.

15. Halwai MA, Mir BA, Dhar SA, et al. Transmalleolar approach to a tubercular lytic lesion of the talar body: a case report J Foot Ankle Surg 2011;50:490-3. 10.1053/j.jfas.2009.02.016

16. Karkhur Y, Tiwari V, Lodhi J, Tiwari A. Astragalus Tuberculosis: A Case Report and Review of the Literature. Cureus 2017;9(9):e1708. doi:10.7759/cureus. 1708 